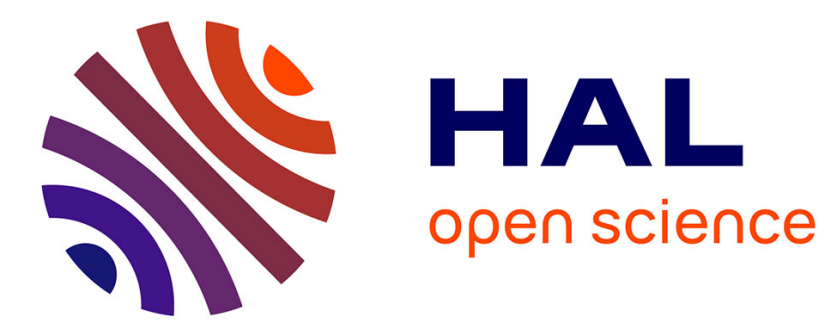

\title{
Unlearning in the BCM learning rule for plastic self-organization in a multi-modal architecture
}

\author{
Mathieu Lefort, Yann Boniface, Bernard Girau
}

\section{To cite this version:}

Mathieu Lefort, Yann Boniface, Bernard Girau. Unlearning in the BCM learning rule for plastic selforganization in a multi-modal architecture. International conference on Artificial Neural Networks -

ICANN 2011, Jun 2011, Espoo, Finland. inria-00585672

\section{HAL Id: inria-00585672 \\ https://hal.inria.fr/inria-00585672}

Submitted on 13 Apr 2011

HAL is a multi-disciplinary open access archive for the deposit and dissemination of scientific research documents, whether they are published or not. The documents may come from teaching and research institutions in France or abroad, or from public or private research centers.
L'archive ouverte pluridisciplinaire HAL, est destinée au dépôt et à la diffusion de documents scientifiques de niveau recherche, publiés ou non, émanant des établissements d'enseignement et de recherche français ou étrangers, des laboratoires publics ou privés. 


\title{
Unlearning in the BCM learning rule for plastic self-organization in a multi-modal architecture
}

\author{
Mathieu Lefort, Yann Boniface, and Bernard Girau \\ LORIA, Campus Scientifique, BP 239, 54506 Vandœuvre-lès-Nancy Cedex, France \\ \{mathieu.lefort, yann.boniface, bernard.girau\}@loria.fr
}

\begin{abstract}
An agent moving in a real environment perceives it by numerous noisy sensors which provide some high dimensionality data with unknown topology. In order to interact in this complex and changing environment, according to the active perception theory, the agent needs to learn the correlations between its actions and the changes they induce in the environment. In the perspective of a bio-inspired architecture for the learning of multi-modal correlations, this article focuses on the ability to forget some previously learned selectivity in a model of perceptive map which spatially codes the sensor data. This perceptive map combines the BCM (Bienenstock Cooper Munro) learning rule, which raises a selectivity to a stimulus, with the neural field (NF) theory, which provides spatial constraints to self-organize the selectivities at the map level. The introduction of an unlearning term in the BCM learning rule (BCMu) improves the BCM-NF coupling by providing plasticity to the self-organization.
\end{abstract}

Keywords: BCM learning rule, dynamic neural fields, self-organization, unlearning, plasticity, multi-modality

\section{Introduction}

Gibson has defined the notion of affordances which corresponds to the possible actions that an agent can perform with an object [1]. An object is then defined by the set of its affordances. The idea that actions take an essential part in the notion of object is also developed by O'Regan and Noë [2], who define an object as a sensory-motor invariant.

Human beings perceive the world by spatially distant sensors. However, their processing are interacting with each other, as illustrated in the ventriloquist effect [3] or in the Mc Gurk effect [4]. Merging the senses allows the brain to form a consistent perception of the world and to reduce the global noise of the sensors. For example, human reaction time is quicker for a consistent audio-visual stimulus than for a visual or an audio stimulus alone [5].

At a mesoscopic level, the cortex shows a generic structure all over its surface, composed of cortical columns (see [6] for an overview). In the functional view of the cortex, it is made up of several areas dedicated to a specific work. Perceptive areas compute a specific sensory flow, providing a spatial coding by 
self-organization, meaning that close neurons are sensitive to close stimuli, as the orientation coding in visual areas [7] or the tonotopic organization in the auditory cortex [8]. Associative areas merge perceptive flows and influence perceptions by feedback. This hierarchical view explains activities observed during the ventriloquist effect [3] but is questioned as multi-modal neurons were recently found in perceptive areas [9].

We have designed a multi-modal architecture to learn sensory-motor contingencies [10]. In this architecture, perceptive maps self-organize to map the sensor data topology in a two dimensional spatial coding, which is influenced to be consistent with the other perceptions. This self-organization is based on the coupling of neural fields with the BCM (Bienenstock Cooper Munro) learning rule. In this article, we focus on the addition of an unlearning term in the $\mathrm{BCM}$ equation to improve the efficiency of the neural field modulation and bring plasticity to the self-organization in order to better adapt to the multi-modal constraints.

In section 2 , we briefly describe the main features of our multi-modal architecture and our perceptive map (for more details refer to $[10,11]$ ). In section 3 , we introduce an unlearning term in the $\mathrm{BCM}$ equation that provides the forgetting of the discriminated stimulus if the modulation is not consistent. We illustrate the self-organization plasticity provided by the unlearning term in the experiment of section 4 .

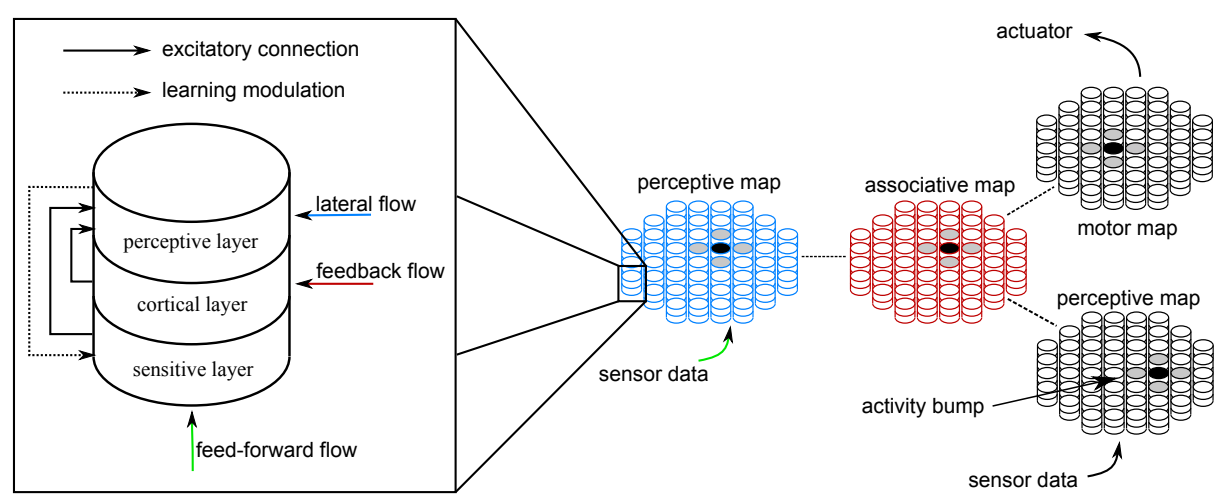

Fig. 1. (right) Example of use of the modular architecture with two sensors and one actuator. (left) Generic architecture of a cortical column in a perceptive map. The sensitive layer receives the feed-forward flow coming from the sensor, the cortical layer is connected to the associative map providing feedback from the multi-modal context and the perceptive layer codes the current perception by an activity bump, resulting from a lateral competition. 


\section{Model}

\subsection{General architecture}

Our architecture consists of interconnected perceptive and associative maps (see figure 1 right). Motor actions are represented by a perceptive map, that corresponds to the related proprioception. All maps have a generic two dimensional structure, to respect the cortex topology, composed of cortical columns with multiple layers. All computations and learning rules have local, continuous, decentralized and unsupervised properties.

Perceptive maps receive a sensory flow and provide a spatially localized activity bump representing the current perception. Sensor data topology is learned with a self-organization mechanism that provides generalization when coupled with the spatial coding. The associative map merges all perceptions to create a multi-modal context that, in return, influences each perception to be consistent. Thus, each perceptive map maps its sensor data flow so that its self-organization is consistent with the other maps. A sensory-motor correlation is represented by the set of the activity bump localizations, that is learned in the weights of the inter map connections.

\subsection{Perceptive map}

Our model of perceptive map is composed of multilayer cortical columns (see figure 1 left). The sensitive layer uses the bio-inspired BCM (Bienenstock Cooper Munro) learning rule [12], which is based on an hebbian rule with a sliding threshold between long term potentiation (LTP) and long term depression (LTD). This LTP/LTD sliding threshold induces competition between inputs, so that, applied to a stimuli flow, this rule has the property to autonomously develop a selectivity to one stimulus. The cortical layer is connected to the associative map and its activity represents the multi-modal influence. The perceptive layer uses neural fields $[13,14]$ to filter its input, corresponding to the cortical and the sensitive informations. The perceptive activity represents the membrane potential of a discrete manifold, which evolves with a differential equation summing the input term with a lateral term, corresponding to an intra map connectivity with a difference of Gaussian shape, and a decay term, to suppress the activity in case of missing input. This lateral connectivity induces spatial competition that raises an activity bump where the input is spatially and temporally consistent. These spatial constraints are propagated to the organization of the sensitive layer, with the modulation of the sensitive activity by the perceptive one.

Thus, the coupling of the sensitive layer with the perceptive layer provides a self-organization of the selectivities of the sensitive layer that maps the sensor data topology. This self-organization can be influenced, by modifying the perceptive activity, to be consistent with the multi-modal context. 


\section{Unlearning}

\subsection{Motivation}

The self-organization of the sensitive layer results from its continuous interaction loop with the perceptive layer. Although, the dynamic appears as a sequential process as the modulation can appear only when the sensitive layer begins to develop a selectivity. The perceptive modulation of the sensitive activity modifies the basins of attraction of the BCM learning rule to favor a specific stimulus. However, solutions of the BCM equation are stable so that the modulation is efficient only if the BCM equation is far from convergence. Thus, the efficiency of the sensitive activity modulation depends on the time of apparition of the perceptive activity (see figure 2).

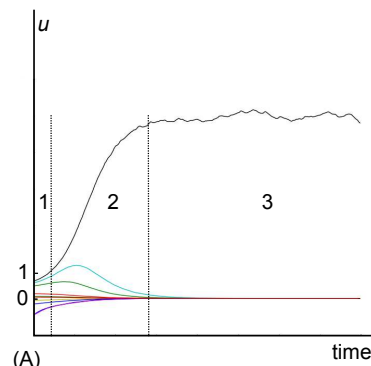

(A)

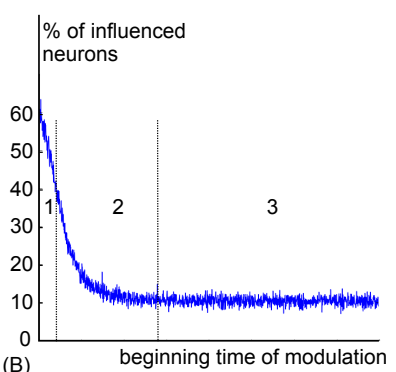

(B)

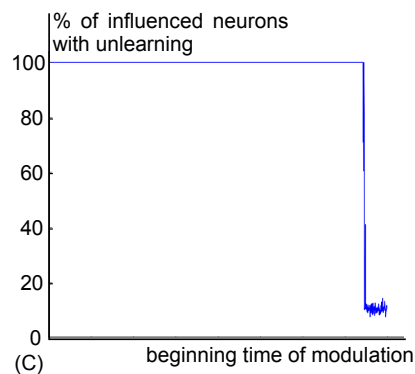

Fig. 2. Compared evolution dynamic of the activity for each stimulus (A), of the percentage of influenced neurons by an additive modulation as a function of its beginning time without unlearning (B) or with unlearning (C). These data are generated with ten orthogonal stimuli with a uniform probability of apparition.

More technically, the dynamic of the BCM learning rule can be split into three steps. In the first phase (1), the neuron have random weights so that activities are similar for each stimulus and the modulation is efficient. During the second phase (2), the threshold value increases and induces competition between inputs. As a consequence, the difference between the response value to the discriminated stimulus and the other one increases, leading to a decreasing efficiency of the modulation. The third phase (3) corresponds to a neuron that has developed a selectivity to the discriminated stimulus with a value equal to the inverse probability of its apparition. This selectivity is stable, and the modulation is no more efficient because of the important and stimulus dependent gap between activities (a neuron has 10\% chance to be influenced, which corresponds to random chance as there are ten uniformly distributed stimuli).

Once the BCM learning rule has converged, this equilibrium is stable, so that the modulation has no more effect on it. This means that at the map level, the 
obtained self-organization is stable whatever the modulation. However, the selforganization needs to be plastic to adapt to the multi-modal constraints. Moreover, these constraints may change over time in case of addition, suppression or deterioration of a perceptive map. The idea is to modify the BCM learning rule, so that the selectivity of a stimulus is no more a stable point if the modulation is not consistent with its selectivity.

\subsection{Equations}

In the BCM learning rule, the activity $u$ of a neuron is equal to the weighted sum of the stimulus $\mathbf{x}$ (equation (1)). The LTP/LTD sliding threshold $\theta$ is computed as the recent expectation of the square of the neuron activity (equation (2)). The weight evolution is based on an hebbian rule using the LTP/LTD threshold (equation (3)).

$$
\begin{aligned}
u & =\mathbf{w} \cdot \mathbf{x} \\
\theta & =E_{\tau}\left[u^{2}\right] \\
\Delta \mathbf{w} & =\eta \mathbf{x} u(u-\theta)
\end{aligned}
$$

To influence the neuron selectivity, its activity is modulated by an increasing function $m$ of the perceptive layer $s$ (equation (4)) [15]. The modulation is additive to be more efficient on non discriminated stimuli, whose values are nearby 0 . We add an unlearning term in the evolution equation of $\mathbf{w}$ (equation (7)). The function $\hat{f}$ is a sigmoid that detects the lack of modulation ( 1 for no modulation and tending to 0 for a high one). Thus, the $\hat{f}(s) u^{2}$ term detects inconsistency between the selectivity and the modulation. $\beta$ is a constant and $\chi$ is the recently expected modulation, so that unlearning is active only if the perceptive layer raises activity bumps. $w \times x$ stands for the term-by-term multiplication of the weight vector with the input vector. Thus, if the neuron is modulated and its selectivity is inconsistent with the modulation, its weights will decrease especially for the current stimulus.

$$
\begin{aligned}
u & =\mathbf{w} \cdot \mathbf{x}+m(s) \\
\theta & =E_{\tau}\left[u^{2}\right] \\
\chi & =E_{\tau^{\prime}}[s] \\
\Delta \mathbf{w} & =\eta\left(\mathbf{x} u(u-\theta)-\beta \chi \hat{f}(s) u^{2} \mathbf{w} \times \mathbf{x}\right)
\end{aligned}
$$

\subsection{Properties}

The $\mathrm{BCM}$ equation with unlearning $(\mathrm{BCMu})$ has three solutions that correspond to a non discriminated stimulus and to a discriminated one with or without consistent modulation. The stability of each nine couples of these three solutions is 
tested by adding a small perturbation of the weights and analyzing its evolution. This mathematical analysis ${ }^{1}$ shows that the only stable points of the $\mathrm{BCMu}$ equation correspond to a selectivity to one stimulus that can be modulated or not. In the case of a modulated stimulus, the unlearning term is equal to 0 so that the stable point is the same as in the BCM learning rule (see [16] for more details). However, in the case of a non modulated stimulus, its value is limited by a fixed value. Thus, this stable point is no more stable if another stimulus is sufficiently modulated because of the competition between stimuli introduced by the LTP/LTD sliding threshold.

Practically, the $\mathrm{BCMu}$ learning rule raises a selectivity to a stimulus, that is forgotten if it does no longer fit the modulation. Thus, the selectivity becomes consistent with the modulation, whenever it appears and shows some spatio-temporal continuity (see figure $2(\mathrm{C})$ ). At the map level, the sensitive self-organization is still stable but is plastic to the changes of the perceptive modulation that represents a consensus between the local sensation and the multi-modal constraints.

\section{Results}

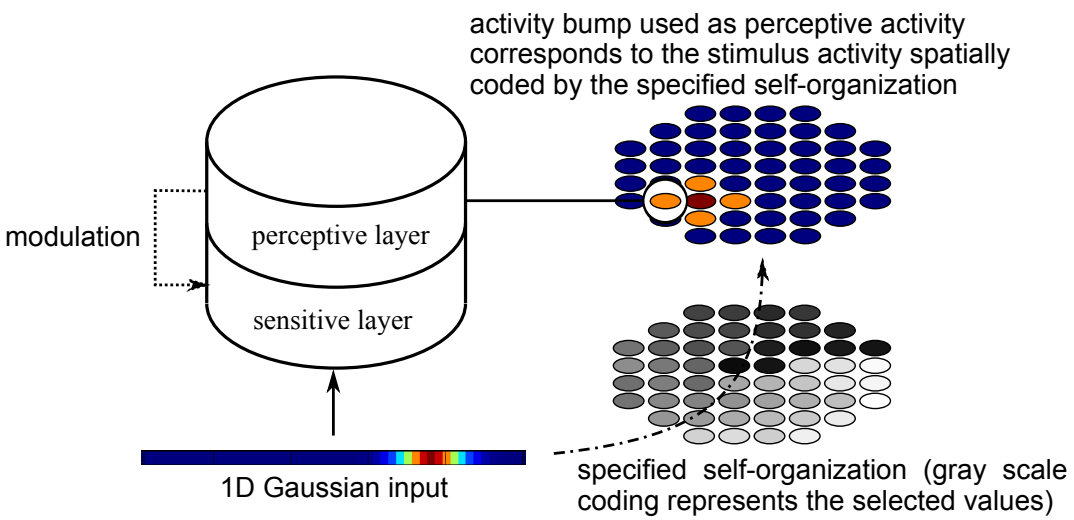

Fig. 3. Protocol used to test the plasticity of the self-organization to the multi-modal constraints. The multi-modal context is the current sensor data spatially coded by a defined self-organization.

The perceptive activity is artificially fixed, independently of the sensitive activity, and is equal to the spatial coding of the current stimulus corresponding to a specified self-organization (see figure 3 ). This activity may represent a consensus between local sensation and multi-modal constraints. This self-organization changes over time, representing a change in multi-modal constraints.

\footnotetext{
${ }^{1}$ see www.loria.fr/ lefortma/recherche/icann/annexes.pdf for the equations
} 
Figure 4 shows the comparative results between the self-organization that determines the perceptive activity and the obtained self-organization of the sensitive layer, using the $\mathrm{BCM}$ or the $\mathrm{BCMu}$ learning rule. The first self-organization is correctly learned by the perceptive map, with or without the unlearning term, meaning that the modulation is efficient on the sensitive self-organization. We can notice that the self-organization is slightly smoother with the use of the unlearning term.

Visually, the self-organization of the sensitive layer without unlearning appears 'frozen', whereas the BCMu rule succeeds in self-organizing the layer in accordance with the modulation changes (see figure 4 (A) second row). The value of the difference with respect to the fixed self-organization remains high when using the BCM rule, whereas it decreases with the BCMu rule until reaching a value close to the one obtained for the first fixed self-organization (see figure $4(\mathrm{~B}))$.

(1)
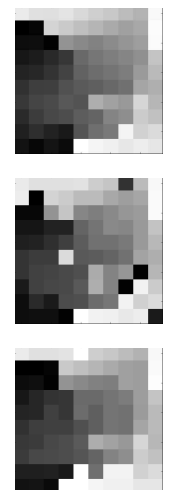

(2)
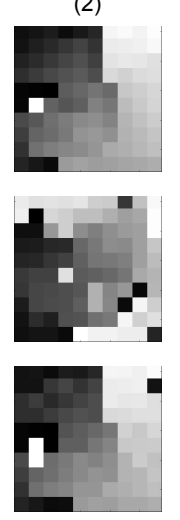

(3)
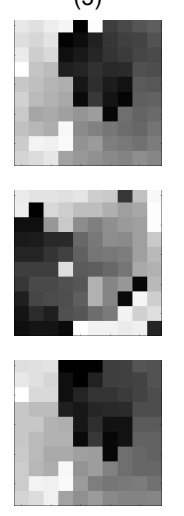

(A)

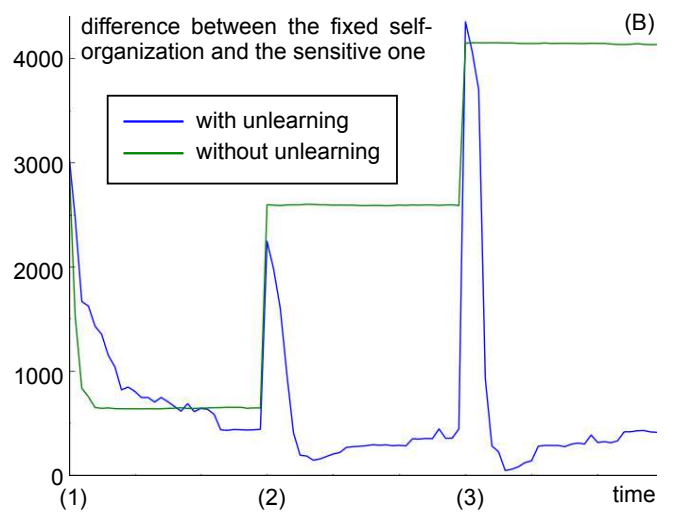

Fig. 4. (A) The selectivity of a column is represented in gray scale. Successive fixed selforganizations that provide the perceptive activity are shown in the first row. Sensitive self-organizations using the BCM learning rule (respectively $\mathrm{BCMu}$ ) are in the second (respectively third) row. (B) Evolution of the sum over all columns of the difference between the selectivities of the fixed self-organization and the sensitive one. The peaks correspond to the change of fixed self-organization.

\section{Conclusion}

This article presents a forgetting mechanism for the BCM learning rule that consists in adding an unlearning term. This term induces a decreasing activity for the discriminated stimulus, if it is not consistent with the modulation received until it is limited by a constant term. Thus, with an efficient modulation, the only stable solution of this learning rule is the discrimination of a single stimulus which is consistent with the received modulation. 
Our model of perceptive map for multi-modal association is based on the coupling of a sensitive layer which raises sensitive information with a perceptive layer which filters this information to raise a spatially localized activity, consistent with the other perceptions. The use of the BCM learning rule with unlearning $(\mathrm{BCMu})$ in our perceptive map model provides plasticity to the selforganization of the sensitive layer. This plasticity is useful to adapt to the multimodal constraints of a changing environment.

\section{References}

1. Gibson, J. J.: The theory of affordances. In: Perceiving, acting, and knowing: Toward an ecological psychology, pages 67-82. 1977.

2. O'Regan, J.K., Noë, A.: A sensorimotor account of vision and visual consciousness. Behavioral and brain sciences, 24(05):939-973, 2001.

3. Bonath, B., Noesselt, T., Martinez, A., Mishra, J., Schwiecker, K., Heinze, H.J., Hillyard, S.A.: Neural basis of the ventriloquist illusion. Current Biology, 17(19):16971703, 2007.

4. Mcgurk, H., Macdonald, J.: Hearing lips and seeing voices. Nature, 264(5588):746748, December 1976.

5. Goldring, J.E., Dorris, M.C., Corneil, B.D., Ballantyne, P.A., Munoz, D.R.: Combined eye-head gaze shifts to visual and auditory targets in humans. Experimental Brain Research, 111(1):68-78, 1996.

6. Mountcastle, V.B.: The columnar organization of the neocortex. Brain, 120(4):701, 1997.

7. Bosking, W.H., Zhang, Y., Schofield, B., Fitzpatrick, D.: Orientation selectivity and the arrangement of horizontal connections in tree shrew striate cortex. Journal of neuroscience, 17(6):2112, 1997.

8. Romani, G.L., Williamson, S.J., Kaufman, L.: Tonotopic organization of the human auditory cortex. Psychiatry, 132:650, 1975.

9. Cappe, C., Barone, P.: Heteromodal connections supporting multisensory integration at low levels of cortical processing in the monkey. European Journal of Neuroscience, 22(11):2886-2902, 2005.

10. Lefort, M., Boniface, Y., Girau, B.: Self-organization of neural maps using a modulated BCM rule within a multi-modal architecture. In: Proceedings of Brain Inspired Cognitive Systems, 2010.

11. Lefort, M., Boniface, Y., Girau, B.: Auto-organisation d'une carte de neurones BCM sous contrainte multimodale. In: Neurocomp, 2010.

12. Bienenstock, E.L., Cooper, L.N., Munro, P.W.: Theory for the development of neuron selectivity: orientation specificity and binocular interaction in visual cortex. Journal of Neuroscience, 2(1): 32, 1982.

13. Amari, S.: Dynamics of pattern formation in lateral-inhibition type neural fields. Biological Cybernetics, 27(2):77-87, 1977.

14. Rougier, N.P., Vitay, J.: Emergence of attention within a neural population. Neural Networks, 19(5):573-581, 2006.

15. Girod, T., Alexandre, F.: Effects of a Modulatory Feedback upon the BCM learning rule. In: BMC Neuroscience, 2009

16. Cooper, L.N.: Theory of cortical plasticity. World Scientific Pub Co Inc, 2004. 